\title{
Mechanism of enhanced light output efficiency in InGaN-based microlight emitting diodes
}

\author{
H. W. Choi, ${ }^{\text {a) }}$ C. W. Jeon, and M. D. Dawson \\ Institute of Photonics, University of Strathclyde, Glasgow, G4 ONW, United Kingdom \\ P. R. Edwards and R. W. Martin \\ Department of Physics, University of Strathclyde, Glasgow, G4 ONG, United Kingdom \\ S. Tripathy \\ Institute of Materials Research and Engineering, 3 Research Link, Singapore 117602
}

(Received 16 December 2002; accepted 21 February 2003)

\begin{abstract}
Micro-light emitting diode (LED) arrays with diameters of 4 to $20 \mu \mathrm{m}$ have been fabricated and were found to be much more efficient light emitters compared to their broad-area counterparts, with up to five times enhancement in optical power densities. The possible mechanisms responsible for the improvement in performance were investigated. Strain relaxation in the microstructures as measured by Raman spectroscopy was not observed, arguing against theories of an increase in internal quantum efficiency due to a reduction of the piezoelectric field put forward by other groups. Optical microscope images show intense light emission at the periphery of the devices, as a result of light scattering off the etched sidewalls. This increases the extraction efficiency relative to broad area devices and boosts the forward optical output. In addition, spectra of the forward emitted light reveal the presence of resonant cavity modes [whispering gallery (WG) modes in particular] which appear to play a role in enhancing the optical output. (C) 2003 American Institute of Physics.
\end{abstract}

[DOI: $10.1063 / 1.1567803$ ]

\section{INTRODUCTION}

$\mathrm{GaN}$ and its alloys have been recognized as important materials for a multitude of applications in the areas of photonic and power electronic devices. ${ }^{1}$ On-going progress in the research and development of GaN-based light-emitting diodes (LEDs) and edge-emitting laser diodes has led to the commercialization of these devices. A range of efforts has been made in the areas of epitaxy, processing, and packaging to improve their optical output characteristics.

The use of microstructures in LEDs has been demonstrated in several reports. In all instances, the light output capabilities of the microstructure devices proved to be superior to their macrosize counterparts. The light output of a photonic device is dependent on its internal quantum efficiency $\left(\eta_{\text {int }}\right)$ and its extraction efficiency $\left(\mathrm{C}_{\mathrm{ex}}\right)$, where $\eta_{\text {int }}$ is an inherent property of the multiquantum well structures in the LED, while the extraction efficiency is greatly dependent on the geometry of the device. Microstructures potentially have an effect on both $\eta_{\text {int }}$ and $\mathrm{C}_{\text {ex }}$ (Ref. ${ }^{2}$ ) among others. For in stance, Dai et al. ${ }^{3}$ proposed that the twofold increment in light output from their $6 \mu \mathrm{m}$ microdisk structures could not be accounted for simply by an increase of $\mathrm{C}_{\mathrm{ex}}$. Based on the observed blue-shift and enhanced intensity photoluminescence peak from their microdisk, it was suggested that a reduction of piezoelectric field strength due to partial strain relief played an active role in the microstructures.

In this article, we report on an investigation into the optical and strain-field properties of GaN-based LEDs with

\footnotetext{
${ }^{a)}$ Electronic mail: anthony.choi@strath.ac.uk
}

micropillar geometry. The mechanism of enhanced light output is examined in detail based on electroluminescence (EL), cathodoluminescence (CL), and Raman scattering results.

\section{EXPERIMENTAL DETAILS}

The LED structure was grown on the $c$-face of a sapphire substrate by metal organic chemical vapor deposition, and consisted of a GaN buffer layer, a $3 \mu \mathrm{m} n$-type GaN layer, and a three-period InGaN/GaN MQW capped with a $0.25 \mu \mathrm{m} p$-type $\mathrm{GaN}$ layer. The pillars, with diameters ranging from 4 to $20 \mu \mathrm{m}$, were patterned onto the LED mesa by inductively coupled plasma etching, of which details have been reported elsewhere. ${ }^{4}$ The bare pillar structures were used for the CL and Raman experiments. A current spreading layer of $30 \mathrm{~nm} \mathrm{Ni} / 30 \mathrm{~nm}$ Au was applied across the mesa to act as $p$ electrodes for the pillar structure to allow for current injection. An optical microphotograph of a fabricated device with $20 \times 2016 \mu \mathrm{m}$ element microLED electrically driven at $20 \mathrm{~mA}$ is shown in Fig. 1.

The Raman spectroscopy measurements were carried out at room temperature using the $514 \mathrm{~nm}$ line of an $\mathrm{Ar}^{+}$laser as an excitation source focused to a spot size of $\sim 1 \mu \mathrm{m}$. The scattered light was detected in the backscattering geometry using a Jobin Yvon T64000 triple-grating spectrometer with a $\mathrm{LN}_{2}$-cooled charge coupled device (CCD) detector. The fabricated microLEDs were characterized in a probe-station configuration. Electroluminescence (EL) data were collected with a CCD detection system $(0.4 \mathrm{~nm}$ resolution), and the output power measurements were performed using a power meter with the Si detector placed in close proximity above the device. The CL measurements were carried out with an 


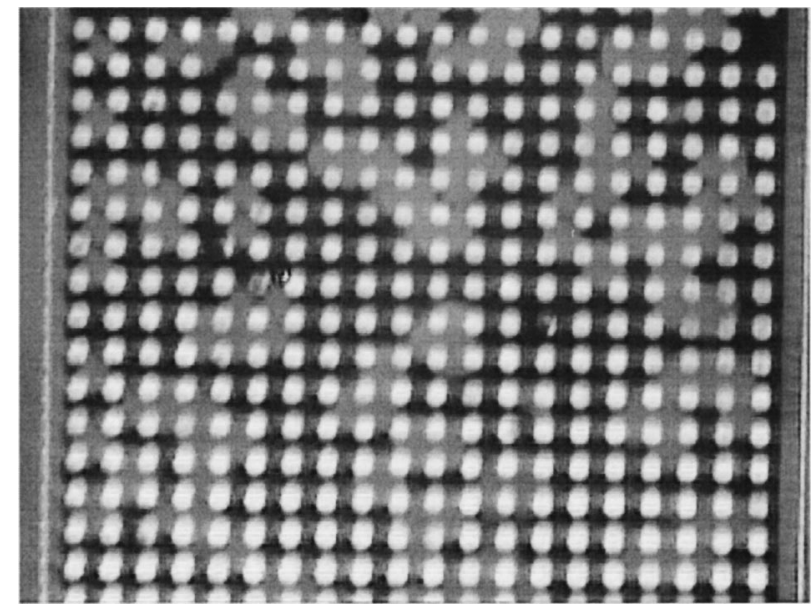

FIG. 1. Optical microphotograph of a fabricated microLED device with pillar dimension of $d=12 \mu \mathrm{m}$, electrically driven at $20 \mathrm{~mA}$.

electron probe microanalyzer (Cameca SX100), with spectra recorded using the same detector as in the EL measurements. Optical images were obtained using a CCD camera mounted on an optical microscope, coaxial with the static electron beam.

\section{RESULTS AND DISCUSSIONS}

The light output characteristics of the devices are shown in Fig. 2. Since the devices have unequal light-generation areas, the power densities are plotted (evaluated by taking the ratio of the actual output powers to their respective total active areas). The device with the smallest pillar dimension, that is, $4 \mu \mathrm{m}$, yields the highest-light output density. Indeed, an enhancement in overall efficiency is observed as the dimensions of the devices are scaled down. Nevertheless, the exact mechanism responsible for the improvement is unknown. We first investigate if this can be attributed to an increase in $\eta_{\text {int }}$. It was explained in Ref. 3 that when the microdisk is smaller than a certain critical size, strain relax-

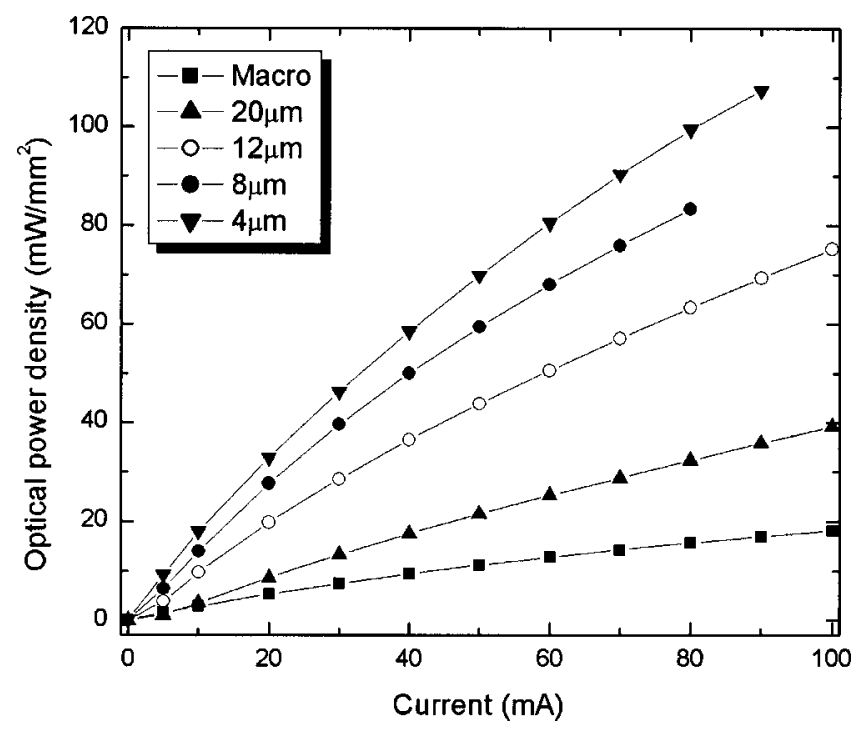

FIG. 2. Normalized optical power output vs current characteristics of microLED devices.

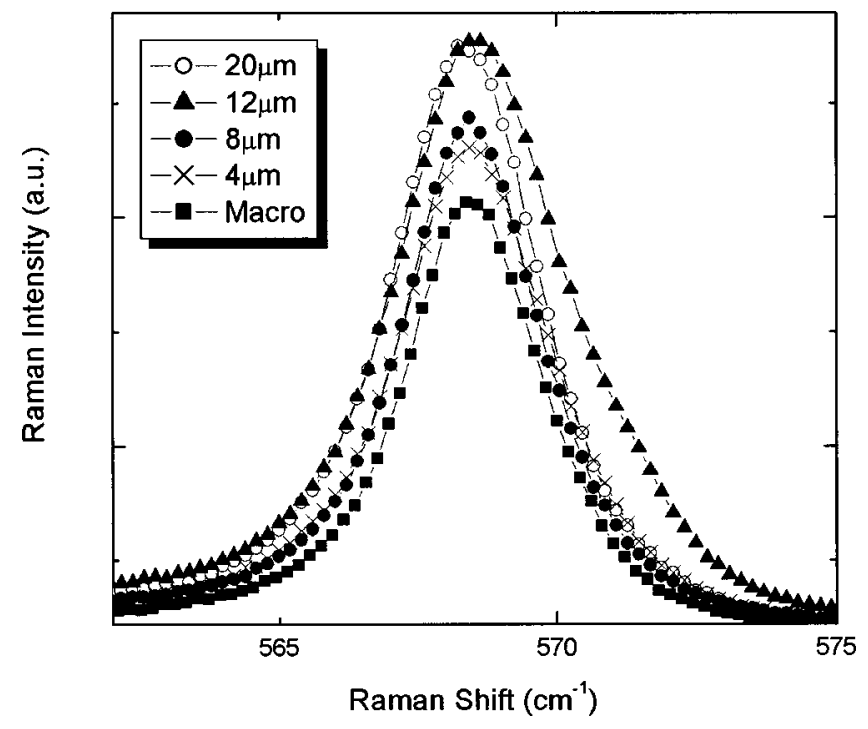

FIG. 3. Raman spectra showing the $E_{2}$ mode taken from the pillar structures.

ation could occur, and hence the piezoelectric field would be reduced, leading to a higher $\eta_{\text {int }}$. Our study will be focused on addressing this consideration in detail.

Demangeot et al. ${ }^{5}$ also reported on strain relaxation in $\mathrm{GaN}$ pillars through Raman spectroscopy experiments. In their work, pillar structures with diameters ranging from 100 $\mathrm{nm}$ to $5 \mu \mathrm{m}$ were formed by reactive ion etching. The $E_{2}$ mode frequency from the Raman spectra was used to monitor the strain component in the structure, since it is much more sensitive to strain compared to other Raman modes due to its independence from the free carrier concentration. On the condition that the electric field of the incident laser line is parallel to the $a$ axis, the $E_{2}$ line will shift towards the higher-frequency region with increasing compressive strain by $2.9 \mathrm{~cm}^{-1} / \mathrm{GPa}^{6}{ }^{6}$ The results in Ref. 4 showed that a significant shift of the $E_{2}$ mode was observed only in pillars with diameters of $1 \mu \mathrm{m}$ and less. Hence, our 4 to $20 \mu \mathrm{m}$ pillars, or the $6 \mu \mathrm{m}$ microdisk described in Ref. 3 , should not experience any strain relaxation.

To verify this point, the pillar structures were subjected to investigations by Raman spectroscopy. In Fig. 3, a set of Raman spectra showing only the $E_{2}$ mode is displayed for the pillars with different diameters, together with that taken from the as-grown material. Not surprisingly, the peak frequencies for all the spectra lie at or near to $568.4 \mathrm{~cm}^{-1}$, characteristic of compressively strained GaN. No significant Raman shift was observed from any of the pillars. We thus conclude, in agreement with Ref. 5, that microstructures of such dimensions (greater than or equal to $4 \mu \mathrm{m}$ ) will not experience any degree of strain relief, and thus that the $\eta_{\text {int }}$ is not dependent on the dimensions of our micro-LEDs.

A blue-shift of the dominant wavelengths in the 4 and 8 $\mu \mathrm{m}$ microLED devices can be observed in Fig. 4. Jin et al. ${ }^{2}$ also reported an observed blue-shift from InGaN microdisk devices, and they explained the phenomenon in terms of partial strain relief. Nevertheless, on the basis of the evidence presented here, we propose that the blue shift is likely to be due to the fact that the devices are driven at different current 


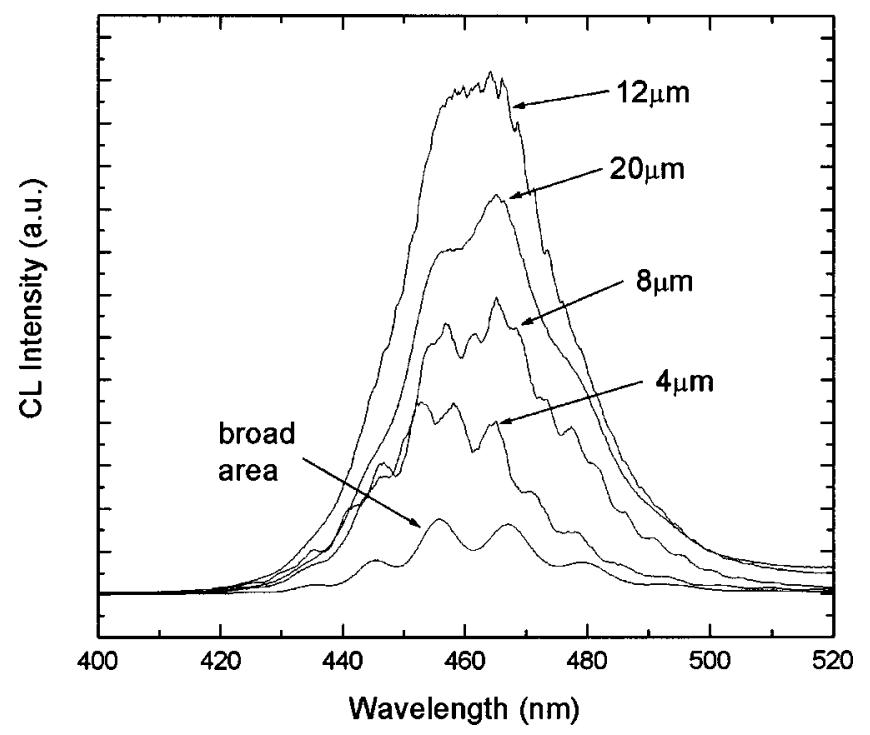

FIG. 4. CL spectra of microLED elements of different diameters, excited by an electron beam of $1 \mu \mathrm{m}$ diameter.

densities. In Fig. 5, we show the EL spectra for a $12 \mu \mathrm{m}$ diameter microLED as a function of injected current ranging from 1 to $40 \mathrm{~mA}$. It can be seen that a significant blue-shift is observed with increasing current. This is attributed to the screening of the piezoelectric field in the quantum wells by the injected carriers. Likewise, the higher-operational current density in the smaller microLEDs enhances the screening effect, thus giving rise to the spectral shift.

CL studies on the device structures are now described. A $20 \mathrm{keV}-1 \mathrm{nA}$ electron beam is focused onto the center of a single pillar to act as an excitation source, giving rise to an

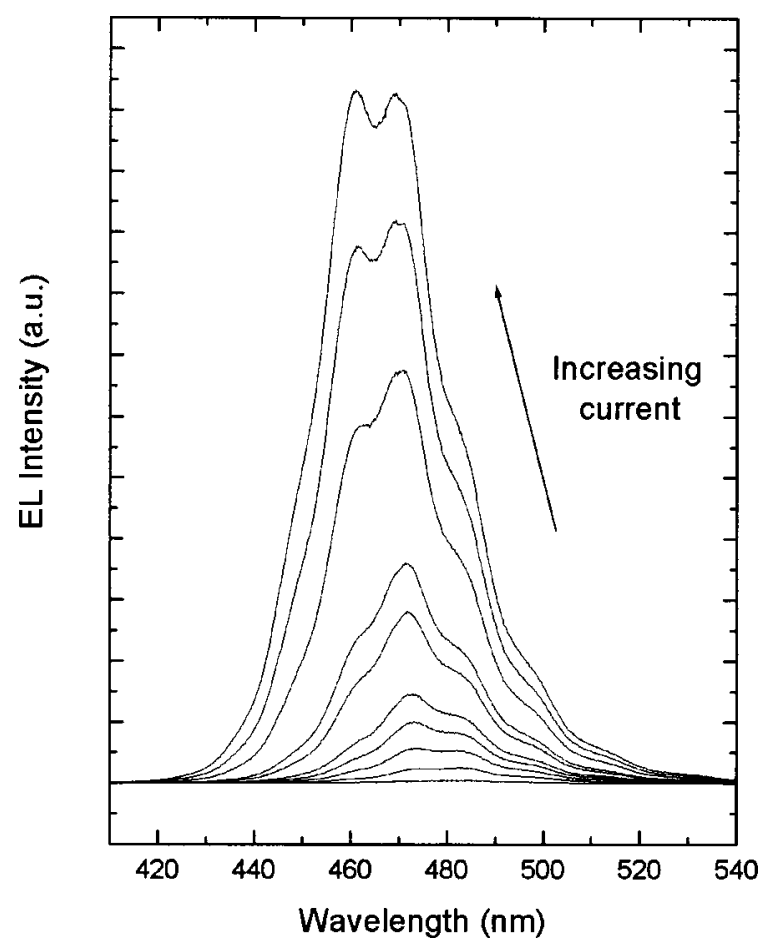

FIG. 5. EL spectra of a single $12 \mu \mathrm{m}$ microLED element driven at different current levels.

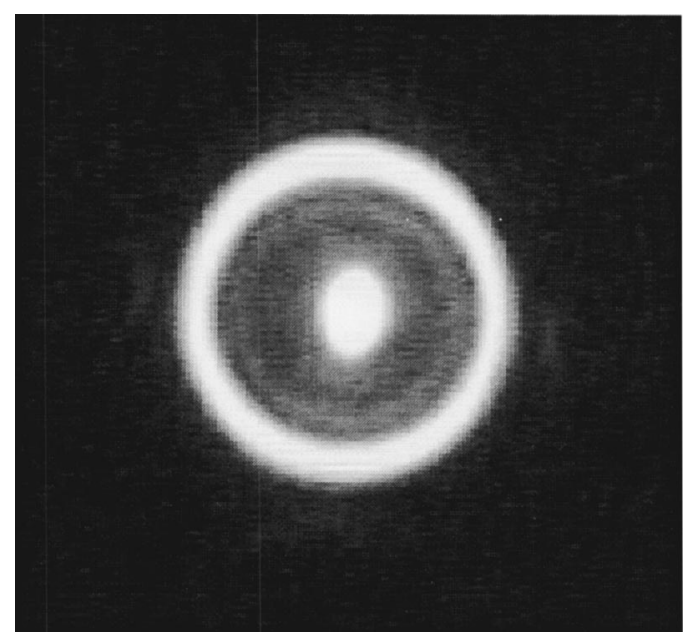

FIG. 6. Opical image of a single $12 \mu \mathrm{m}$ microLED element excited by a focused electron beam.

excitation volume that approximates to a sphere of $1 \mu \mathrm{m}$ diameter. An identical light generation volume in the pillars of different diameters is expected. From the CL spectra shown in Fig. 4, the CL intensity scales according to the pillar diameter (except for $d=20 \mu \mathrm{m}$ ). Having concluded that $\eta_{\text {int }}$ is not affected in structures of such dimensions, the total amount of light generated should be of the same level. Nevertheless, in the current configuration, the detector is placed above the device under test such that only light emitted in the forward direction can be detected. Hence, devices of pillar geometries are able to provide an enhanced level of light output in the forward direction, which is often the direction where the light is most useful.

An optical image of a $12 \mu \mathrm{m}$ pillar excited by a static electron beam is shown in Fig. 6. Apart from a luminescence spot in the center of the pillar corresponding to the site of the excitation beam, a bright luminescence ring is observed at the periphery of the pillar. Much of the in-plane propagating light will be emitted through the sidewall surface, but due to scattering on the etched sidewall, a certain fraction of the light can be extracted from the top surface near the periphery. This accounts for the ring of light observed in the CL images, and also for the greater magnitude of light output in the forward direction. Of course, light reflection off the sidewall is not restricted to circular geometries; the intensity of light emitted near the periphery of a macroLED would also be higher than that emitted at the center. Nevertheless, microLEDs take full advantage of this phenomenon because of the presence of resonant modes, and also due to the fact that the bright ring of light occupies a great fraction of the total pillar surface area. This can be explained in terms of the geometry of the cavity. A microdisk cavity can support two different resonant modes, namely, radial and whispering gallery (WG) modes. ${ }^{7-9}$ Radial modes are dominated by photon wave motion along the radial direction of the disk, the equivalent cavity being formed between the edge and center of the pillar. The WG mode, on the other hand, may be thought of as in-plane light propagation around the inside perimeter of the disk, facilitated by total internal reflection. 


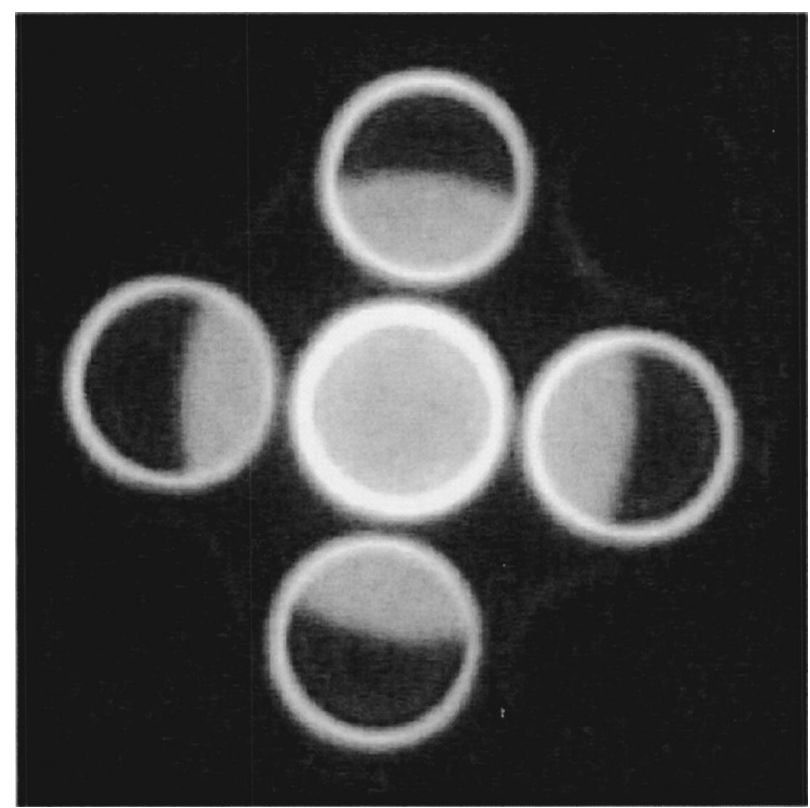

FIG. 7. Optical image of several $12 \mu \mathrm{m}$ microLED elements excited by a defocused $40 \mu \mathrm{m} e$ beam.

We also acknowledge the losses introduced by the etched sidewall, but these are outweighed by the benefits provided by the sidewalls.

The resonant modes, together with interference fringes, results in evenly spaced peaks in the CL spectra, as shown in Fig. 5. The modal spacings ${ }^{9}$ can be evaluated as

$$
\Delta \lambda_{\mathrm{rad}}=\frac{\lambda^{2}}{2 R n},
$$

and

$$
\Delta \lambda_{\mathrm{WG}}=\frac{\lambda^{2}}{2 \pi R n},
$$

where $R$ is the radius of the microLEDs. Modal spacings of $\sim 6.8,3.4$, and $2.3 \mu \mathrm{m}$ were evaluated for the 4,8 , and 12 $\mu \mathrm{m}$ pillar structures, respectively. This is consistent with the theoretical prediction of Eq. (2), confirming the presence of WG modes in the detected light. In fact, the presence of WG modes in a GaN microdisk structure has been reported to reduce the pump intensity for a spontaneous-to-stimulated emission transition by a factor of 10 compared to a $\mathrm{GaN}$ sample without a cavity structure ${ }^{10}$ Although radial modes are not recorded, their presence in the microLED structure cannot be ruled out. Since radial modes have minima at the edge of the structure, scattering off the sidewall is less likely.

As mentioned earlier, peculiarities were observed in the spectra of the $20 \mu \mathrm{m}$ pillar. Its CL spectra appear to be smoother than other spectra, showing little signs of resonant modes. Also, the CL intensity is lower than that of the $12 \mu \mathrm{m}$ pillar. This can be attributed to absorption, since the absorption coefficient of $\mathrm{GaN}$ at this wavelength is approximately $10^{3} \mathrm{~cm}^{-1},{ }^{11}$ corresponding to a mean absorption length of 10 $\mu \mathrm{m}$. A large fraction of the in-plane propagating light is absorbed before reaching the sidewalls. This also applies to the
WG modes, where light has to travel longer distances around the circumference in the $20 \mu \mathrm{m}$ pillar. Hence, the intensity of the in-plane light at the periphery will be lower compared to small diameter pillars.

We have presented the case for microsized pillars excited by a point source. In a current injection device, radiative processes occur throughout the active area. We simulate this case by defocusing the excitation beam source to a diameter of $40 \mu \mathrm{m}$. In Fig. 7, we show the optical image of the same $20 \mu \mathrm{m}$ microLED, this time under large-area stationary electron beam excitation. We note that a bright ring of light is still observable from the periphery of the devices, on top of uniform light emission across the top of the pillar, evidencing the sidewall scattering enhanced by the presence of WG modes. An enhanced level of light can be extracted in the forward direction. The overall extraction efficiency of the devices is also increased compared to large-area devices, as photons have more avenues to escape, and the likelihood of absorption is reduced.

\section{CONCLUSIONS}

In summary, InGaN-based micro-LEDs have been fabricated and characterized. These devices were found to have much higher-light output intensities in the forward direction compared to their macrosized counterparts. From the Raman spectroscopy data, an enhancement of $\eta_{\text {int }}$ is unlikely since strain relaxation was not observed. Instead, the superior light output characteristics can be attributed to the presence of resonant cavity modes (WG modes in particular). These inplane propagating photons may be extracted through the sidewall to give a lateral light output component. On the other hand, through scattering from the etched sidewall, some photons may be emitted from the top near the periphery of the mesa, which appears as a ring of light surrounding the pillar.

\section{ACKNOWLEDGMENTS}

The authors would like to thank A. C. Bryce and R. M. De La Rue of Glasgow University for support of metal deposition carried out in this work.

${ }^{1}$ S. Nakamura and G. Fasol, The Blue Laser Diode (Springer, New York, 1997).

${ }^{2}$ S. X. Jin, J. Li, J. Y. Lin, and H. X. Jiang, Appl. Phys. Lett. 77, 3236 (2000).

${ }^{3}$ L. Dai, B. Zhang, J. Y. Lin, and H. X. Jiang, J. Appl. Phys. 89, 4951 (2001)

${ }^{4}$ R. W. Martin, P. R. Edwards, H. S. Kim, K. S. Kim, T. Kim, I. M. Watson, M. D. Dawson, Y. Cho, T. Sands, and N. W. Cheung, Appl. Phys. Lett. 79, 3029 (2001)

${ }^{5}$ F. Demangeot, J. Gleize, J. Frandon, M. A. Renucci, M. Kuball, D. Peyrade, L. Manin-Ferlazzo, Y. Chen, and N. Grandjean, J. Appl. Phys. 91, 6520 (2002)

${ }^{6}$ F. Demangeot, J. Frandon, M. A. Renucci, O. Briot, B. Gil, and R. L. Aulumbard, Solid State Commun. 100, 207 (1996).

${ }^{7}$ A. Onischenko and J. Sarma, IEE Proc.: Optoelectron. 143, 67 (1996).

${ }^{8}$ R. A. Mair, K. C. Zeng, J. Y. Lin, H. X. Jiang, B. Zhang, L. Dai, A. 
Botchkarev, W. Kim, H. Morkoc, and M. A. Khan, Appl. Phys. Lett. 72, 1530 (1998).

${ }^{9}$ K. S. Kim, P. R. Edwards, H. S. Kim, R. W. Martin, I. M. Watson, and M. D. Dawson, Phys. Status Solidi A 228, 169 (2001).
${ }^{10}$ S. Chang, N. B. Rex, R. K. Chang, G. Chong, and L. J. Guido, Appl. Phys. Lett. 75, 166 (1999).

${ }^{11}$ O. Ambacher, W. Rieger, P. Ansmann, H. Angerer, T. D. Moustakas, and M. Stutzmann, Solid State Commun. 97, 365 (1996). 